\title{
COMMON FIXED POINT THEOREMS WITH AN APPLICATION IN DYNAMIC PROGRAMMING
}

\author{
GUOJING JIANG AND SHIN MIN KANG
}

Received 21 April, 2017

\begin{abstract}
Two common fixed point theorems for a class of contractive mappings are proved in metric spaces. As an application, the existence and uniqueness of solution for a functional equation arising in dynamic programming is given. The results presented in this paper generalize some known results in the literature.
\end{abstract}

2010 Mathematics Subject Classification: 54H25; 49L20; $90 \mathrm{C} 39$

Keywords: common fixed point, class of contractive mappings, diminishing orbital diameters, functional equation, dynamic programming

\section{INTRODUCTION AND PRELIMINARIES}

Throughout this paper, unless otherwise stated, $(X, d)$ denotes a metric space. Let $\mathbb{R}=(-\infty,+\infty), \mathbb{R}^{+}=[0,+\infty)$, and for each $t \in \mathbb{R},[t]$ denote the greatest integer not exceeding $t$. Let $\omega$ and $\mathbb{N}$ denote the sets of all nonnegative integers and positive integers, respectively, and

$$
\begin{aligned}
& \Phi=\left\{\varphi: \varphi: \mathbb{R}^{+} \rightarrow \mathbb{R}^{+} \text {is upper semi-continuous and nondecreasing and } \phi(t)<t\right. \\
& \text { for } t>0\} .
\end{aligned}
$$

Let $f$ be a self mapping of $(X, d)$. For $x, y \in X$ and $A, B \subseteq X$, define

$$
\begin{aligned}
O(x, f) & =\left\{f^{n} x: n \in \omega\right\}, \quad O(x, y, f)=O(x, f) \cup O(y, f), \\
C_{f} & =\{g: g \text { is a self mapping of } X \text { and } g f=f g\}, \\
\delta(A, B) & =\sup \{d(a, b): a \in A, b \in B\}, \quad \delta(A)=\delta(A, A) .
\end{aligned}
$$

It is easy to see that $\left\{f^{n}: n \in \omega\right\} \subseteq C_{f}$.

Many authors studied the existence and uniqueness of fixed point and common fixed point for several classes of contractive mappings and families of contractive mappings in metrics spaces, and they used a few fixed point and common fixed point theorems to establish the existence and uniqueness of solution and common solutions for some kinds of functional equations and systems of functional equations arising 
from dynamic programming, for example, see [1-25] and the references therein. Jungck [9] proved some fixed point theorems for $C_{f}$ in metric spaces. Ohta and Nikaido [21] established two fixed point theorems for contractive mappings which satisfy

$$
d\left(f^{k} x, f^{k} y\right) \leq \alpha \delta(O(x, y, f)), \quad \forall x, y \in X,
$$

where $\alpha \in[0,1)$.

As suggested in Bellman and Lee [1], the basic form of the functional equations in dynamic programming is

$$
f(x)=\max _{y \in S} H(x, y, f(T(x, y))), \quad \forall x \in D,
$$

where $x$ and $y$ denote the state and decision vectors, respectively, $T$ denotes the transformation of the process and $f(x)$ denotes the optimal return function with the initial state $x$.

Motivated by the results in [1-25], in this paper we extend the classes of mappings (1.1) and functional equations (1.2) considered by Ohta and Nikaido [21] and Bellman and Lee [1], respectively, to the following more general classes of contractive mappings and functional equations:

$$
d\left(f^{p} x, f^{q} y\right) \leq \varphi\left(\delta\left(\cup_{g \in C_{f}} g O(x, y, f)\right)\right), \quad \forall x, y \in X,
$$

where $\varphi \in \Phi$ and $p, q$ are some positive integers, and

$$
f(x)=\underset{y \in D}{\operatorname{opt}}\{u(x, y)+H(x, y, f(T(x, y)))\}, \quad \forall x \in S,
$$

where $u: S \times D \rightarrow \mathbb{R}, T: S \times D \rightarrow S$ and $H: S \times D \times \mathbb{R} \rightarrow \mathbb{R}$, the opt denotes max or min. Under certain conditions we study the existence and uniqueness of fixed point, common fixed point and solution for the contractive mapping (1.3), the family of mappings $C_{f}$ and the functional equation (1.4), respectively, and establish the convergence and error estimates of Picard iterations with respect to the fixed point of the contractive mapping (1.3) and the solution of the functional equation (1.4), respectively. The results presented in this paper extend and improve some known results in $[7,21]$.

Let us recall the following notation, definitions and lemmas.

Definition 1 ([2]). Let $(X, d)$ be a metric space. A mapping $f: X \rightarrow X$ is said to have diminishing orbital diameters in $X$ if

$$
\lim _{n \rightarrow \infty} \delta\left(O\left(f^{n} x, f\right)\right)<\delta(O(x, f)) \quad \text { for all } x \in X \text { with } \delta(O(x, f))>0 .
$$

Definition 2 ([6]). Let $(X, d)$ be a metric space, $A \subseteq X$ and $A_{n} \subseteq X$ for $n \in \mathbb{N}$. The sequence $\left\{A_{n}\right\}_{n \in \mathbb{N}}$ is said to converge to $A$ if

(1) each point $a \in A$ is the limit of some convergent sequence $\left\{a_{n}: a_{n} \in A_{n}\right.$ for each $n \in \mathbb{N}\}$

(2) for arbitrary $\epsilon>0$, there exists $k \in \mathbb{N}$ such that $A_{n} \subseteq A_{\epsilon}$ for $n>k$, where $A_{\epsilon}$ is the union of all open spheres with centers in $A$ and radius $\epsilon$. 
Lemma 1 ([24]). Let $\varphi \in \Phi$ and $\varphi^{n}$ denote the composition of $\varphi$ with itself $n$ times. Then for every $t>0, \varphi(t)<t$ if and only if $\lim _{n \rightarrow \infty} \varphi^{n}(t)=0$.

Lemma 2 ([20]). Let $f$ a continuous self mapping of a metric space $(X, d)$ such that

(a) $f$ has a unique fixed point $u \in X$;

(b) $\lim _{n \rightarrow \infty} f^{n} x=u$ for all $x \in X$;

(c) there exists an open neighborhood $G$ of $u$ with the property that given any open set $V$ containing $u$ there exists $k \in \mathbb{N}$ such that $f^{n} G \subseteq V$ for all $n>k$.

Then for any $r \in(0,1)$, there exists a metric $d^{\prime}$, topologically equivalent to $d$, such that $d^{\prime}(f x, f y) \leq r d^{\prime}(x, y)$ for all $x, y \in X$.

\section{COMMON FIXED POINT THEOREMS FOR $C_{f}$}

Our main results are as follows.

Theorem 1. Let $(X, d)$ be a bounded complete metric space and $f: X \rightarrow X$ satisfy (1.3). Assume that $f$ is continuous. Then

(i) $f$ has diminishing orbital diameters in $X$;

(ii) $f$ has a unique fixed point $u \in X$, which is also a unique common fixed point of $C_{f}$;

(iii) $d\left(f^{n} x, u\right) \leq \varphi^{[n / s]}(\delta(X))$ for all $x \in X$ and $n \in \mathbb{N}$, where $s=\max \{p, q\}$;

(iv) $\lim _{n \rightarrow \infty} f^{n} x=u$ for all $x \in X$ and $\left\{f^{n} X\right\}_{n \in \mathbb{N}}$ converges to $\{u\}$;

(v) for any $r \in(0,1)$, there exists a metric $d^{\prime}$, topological equivalent to $d$, such that $d^{\prime}(f x, f y) \leq r d^{\prime}(x, y)$ for all $x, y \in X$.

Proof. We may assume, without loss of generality, $p \geq q$. For any $x, y \in X$ and $n \geq p$, it follows from (1.3) that

$$
\begin{aligned}
d\left(f^{n} x, f^{n} y\right) & =d\left(f^{p} f^{n-p} x, f^{q} f^{n-q} y\right) \\
& \leq \varphi\left(\delta\left(\cup_{g \in C_{f}} g\left(f^{n-p} x, f^{n-q} y, f\right)\right)\right) \\
& =\varphi\left(\delta\left(\cup_{g \in C_{f}} O\left(f^{n-p} g x, f^{n-q} g y, f\right)\right)\right) \\
& \leq \varphi\left(\delta\left(O\left(f^{n-p} X \cup f^{n-q} X\right)\right)\right) \\
& =\varphi\left(\delta\left(f^{n-p} X\right)\right),
\end{aligned}
$$

which implies that

$$
\delta\left(f^{n} X\right) \leq \varphi\left(\delta\left(f^{n-p} X\right)\right), \quad \forall n \geq p .
$$

It follows from (2.1) and Lemma 1 that

$$
\delta\left(f^{k p} X\right) \leq \varphi\left(\delta\left(f^{(k-1) p} X\right)\right) \leq \cdots \leq \varphi^{k}(\delta(X)) \rightarrow 0 \quad \text { as } k \rightarrow \infty .
$$

Because

$$
X \supseteq f X \supseteq f^{2} X \supseteq \cdots \supseteq f^{n} X \supseteq f^{n+1} X \supseteq \cdots, \quad \forall n \in \omega,
$$


by (2.2) we infer that

$$
\delta\left(f^{n} X\right) \rightarrow 0 \quad \text { as } n \rightarrow \infty .
$$

In view of (2.3), we get that

$$
d\left(f^{n} x, f^{n+h} x\right) \leq \delta\left(O\left(f^{n} x, f\right)\right) \leq \delta\left(f^{n} X\right), \quad \forall x \in X, \forall n, h \in \mathbb{N}
$$

It follows from (2.3) and (2.4) that $f$ has diminishing orbital diameters and for each $x \in X,\left\{f^{n} x\right\}_{n \in \mathbb{N}}$ is a Cauchy sequence. Thus $\left\{f^{n} x\right\}_{n \in \mathbb{N}}$ converges to some point $u \in X$ by completeness of $X$.

Since $f$ is continuous, it follows that $f u=u$. Suppose that $f$ has a second fixed point $v \in X$. From (2.3) we have

$$
d(u, v) \leq \delta\left(f^{n} X\right) \rightarrow 0 \quad \text { as } n \rightarrow \infty,
$$

which means that $u=v$, that is, $f$ has a unique fixed point $u$. Let $g \in C_{f}$. Note that $g u=g f u=f g u$. It follows that $g u$ is a fixed point of $f$. Therefore $g u=u$. It follows from $f \in C_{f}$ that $u$ is a unique common fixed point of $C_{f}$. Using (2.1) and (2.3) we conclude that for $x \in X$

$$
\begin{aligned}
d\left(f^{n} x, u\right) & \leq \delta\left(f^{n} X,\{u\}\right) \leq \delta\left(f^{n} X\right) \\
& \leq \varphi^{[n / p]}\left(\left\{\delta\left(f^{i} X\right): 0 \leq i<p\right\}\right) \\
& =\varphi^{[n / p]}(\delta(X)), \quad \forall n \in \mathbb{N} .
\end{aligned}
$$

Given $\epsilon>0$. (2.3) ensures that there exists $k \in \mathbb{N}$ such that $\delta\left(f^{n} X\right)<\frac{\epsilon}{2}$ for $n>k$. Consequently, by (2.4) we deduce that $f^{n} X \subseteq B(u, \epsilon)=\{x \in X: d(u, x)<\epsilon\}$ for $n>k$. Thus $\left\{f^{n} X\right\}_{n \in \mathbb{N}}$ converges to $\{u\}$.

To show (v), it suffices to show that (c) of Lemma 2 holds. Take $G=X$. For any open set $V$ containing $u$ there exists $\epsilon>0$ with $B(u, \epsilon)=\{x \in X: d(u, x)<\epsilon\} \subseteq V$. It follows from what we have just proved that $f^{n} G \subseteq B(u, \epsilon) \subseteq V$ for $n>k$. Hence (c) is satisfied. This completes the proof.

Remark 1. The following example shows that the condition that $f$ be continuous when $p, q>1$ is necessary in Theorem 1 .

Example 1. Let $X=[0,1]$ with the usual metric. Define a discontinuous mapping $f: X \rightarrow X$ by $f 0=1$ and $f x=\frac{x}{2}$ for $x \in(0,1]$. Take $p=2, q=3$ and $\varphi(t)=\frac{t}{2}$ for $t \geq 0$. It is easy to check that the conditions of Theorem 1 are satisfied except for the continuity assumption. $f$ however has no fixed point in $X$.

Remark 2. The following example reveals that the boundedness of $X$ is necessary in Theorem 1.

Example 2. Let $X=[1,+\infty)$ with the usual metric. Define a mapping $f: X \rightarrow X$ by $f x=2 x$ for $x \in X$. Set $p=2, q=3$ and $\varphi(t)=\frac{t}{2}$ for $t \geq 0$. It is easily proved that the conditions of Theorem 1 are satisfied except for the boundedness assumption. $f$ however has no fixed point in $X$. 
Replacing the boundedness of $X$ by the boundedness of $f X$, as in the proof of Theorem 1, we have

Theorem 2. Let $(X, d)$ be a complete metric space and $f: X \rightarrow X$ be a continuous mapping such that $f X$ is bounded and (1.3) holds. Then (i), (ii), (iv) and (v) in Theorem 1 and the following

(iii) $^{\prime} d\left(f^{n} x, u\right) \leq \varphi^{[n / s]}(\delta(f X))$ for all $x \in X, n \in \mathbb{N}$ hold.

Remark 3. Theorems 1 and 2 extend, improve and unify the corresponding results in $[7,21]$.

\section{AN APPLICATION IN DYNAMIC PROGRAMMING}

Throughout this section, we assume that $X, Y$ are Banach spaces, $S \subseteq X$ is the state space, $D \subseteq Y$ is the decision space, $B(S)$ denotes the set of all bounded realvalued functions on $S$ and $d(f, g)=\sup \{|f(x)-g(x)|: x \in S\}$ for $f, g \in B(S)$. Clearly $(B(S), d)$ is a complete metric space.

Theorem 3. Suppose that the following conditions are satisfied:

(a) $u$ and $H$ are bounded;

(b) $|H(x, y, g(t))-H(x, y, h(t))| \leq \varphi\left(\delta\left(\cup_{m \in C_{A}} m O(g, h, A)\right)\right)$ for all $(x, y) \in$ $S \times D, g, h \in B(S)$ and $t \in S$, where $\varphi \in \Phi$ and the mapping $A: B(S) \rightarrow$ $B(S)$ defined by

$$
A g(x)=\underset{y \in D}{\operatorname{opt}}\{u(x, y)+H(x, y, g(T(x, y)))\}, \quad \forall(x, g) \in S \times B(S)
$$

satisfies

(c) For any sequence $\left\{h_{n}\right\}_{n \in \mathbb{N}} \subseteq B(S)$ and $h \in B(S)$,

$$
\lim _{n \rightarrow \infty} \sup _{x \in S}\left|h_{n}(x)-h(x)\right|=0 \Longrightarrow \lim _{n \rightarrow \infty} \sup _{x \in S}\left|A h_{n}(x)-A h(x)\right|=0 .
$$

Then

(i) A has diminishing orbital diameters in $B(S)$;

(ii) the functional equation (1.4) possesses a unique solution $v \in B(S)$, which is both a unique fixed point of $A$ and a unique common fixed point of $C_{A}$;

(iii) $d\left(A^{n} x, v\right) \leq \varphi^{[n / s]}(\delta(A B(S)))$ for all $x \in B(S), n \in \mathbb{N}$;

(iv) $\lim _{n \rightarrow \infty} A^{n} x=v$ for all $x \in B(S)$ and $\left\{A^{n} B(S)\right\}_{n \in \mathbb{N}}$ converges to $\{v\}$;

(v) for any $r \in(0,1)$, there exists a metric $d^{\prime}$, topological equivalent to $d$, such that $d^{\prime}(A x, A y) \leq r d^{\prime}(x, y)$ for all $x, y \in B(S)$.

Proof. It follows from (a), (c) and (3.1) that $A B(S)$ is bounded and $A$ is continuous. We assume that without loss of generality opt $=$ inf. For any $\varepsilon>0, x \in S$ and $h, g \in B(S)$, there exist $y, z \in D$ such that

$$
A g(x)>u(x, y)+H(x, y, g(T(x, y)))-\varepsilon,
$$




$$
A h(x)>u(x, z)+H(x, z, h(T(x, z)))-\varepsilon .
$$

Also we have

$$
\begin{aligned}
& A g(x) \leq u(x, z)+H(x, z, g(T(x, z))), \\
& A h(x) \leq u(x, y)+H(x, y, h(T(x, y))) .
\end{aligned}
$$

From (3.2), (3.5) and (b) we infer that

$$
\begin{aligned}
A g(x)-A h(x) & >H(x, y, g(T(x, y)))-H(x, y, h(T(x, y)))-\varepsilon \\
& \geq-\varphi\left(\delta\left(\cup_{m \in C_{A}} m O(g, h, A)\right)\right)-\varepsilon .
\end{aligned}
$$

Similarly, from (3.3) and (3.4) and (b) we know that

$$
\begin{aligned}
A g(x)-A h(x) & <H(x, z, g(T(x, z)))-H(x, z, h(T(x, z)))+\varepsilon \\
& \leq \varphi\left(\delta\left(\cup_{m \in C_{A}} m O(g, h, A)\right)\right)+\varepsilon .
\end{aligned}
$$

It is easy to see that

$$
\begin{aligned}
d(A g, A h) & =\sup _{x \in S}|A g(x)-A h(x)| \\
& \leq \varphi\left(\delta\left(\cup_{m \in C_{A}} m O(g, h, A)\right)\right)+\varepsilon .
\end{aligned}
$$

Letting $\varepsilon$ tend to zero, we have

$$
d(A g, A h) \leq \varphi\left(\delta\left(\cup_{m \in C_{A}} m O(g, h, A)\right)\right), \quad \forall g, h \in B(S) .
$$

Thus Theorem 3 follows from Theorem 2 with $p=q=1$. Particularly, the unique fixed point $v \in B(S)$ of $A$ is a unique solution of the functional equation (1.4) in $B(S)$. This completes the proof.

\section{REFERENCES}

[1] R. Bellman and E. S. Lee, "Functional equations in dynamic programming," Aequationes Math., vol. 17, no. 1, pp. 1-18, 1978, doi: 10.1007/BF01818535.

[2] L. P. Belluce and W. A. Kirk, "Fixed-point theorems for certain classes of nonexpansive mappings," Proc. Amer. Math. Soc., vol. 20, no. 1, pp. 141-146, 1969, doi: 10.1090/S0002-99391969-0233341-4.

[3] P. C. Bhakta and S. R. Choudhury, "Some existence theorems for functional equations arising in dynamic programming, II,” J. Math. Anal. Appl., vol. 131, no. 1, pp. 217-231, 1988, doi: 10.1016/0022-247X(88)90201-6.

[4] P. C. Bhakta and S. Mitra, "Some existence theorems for functional equations arising in dynamic programming," J. Math. Anal. Appl., vol. 98, no. 2, pp. 348-362, 1984, doi: 10.1016/0022247X(84)90254-3.

[5] S.-S. Chang and Y.-H. Ma, "Coupled fixed points for mixed monotone condensing operators and an existence theorem of the solutions for a class of functional equations arising in dynamic programming," J. Math. Anal. Appl., vol. 160, no. 2, pp. 468-479, 1991, doi: 10.1016/0022247X(91)90319-U.

[6] Deepmala, "Existence theorems for solvability of a functional equation arising in dynamic programming," Int. J. Math. Math. Sci., vol. 2014, no. ARTICLE ID 706585, pp. 1-9, 1984, doi: 10.1155/2014/706585. 
[7] B. Fisher, "Common fixed points of mappings and set-valued mappings," Rostock Math. Kolloq, vol. 18, pp. 69-77, 1981.

[8] G. Jiang, S. M. Kang, and Y. C. Kwun, "Solvability and algorithms for functional equations originating from dynamic programming," Fixed Point Theory Appl., vol. 2011, no. ARTICLE ID 701519, pp. 1-30, 2011, doi: 10.1155/2011/701519.

[9] G. Jungck, "Fixed points for commuting and compatible maps on compact," Proc. Amer. Math. Soc., vol. 103, no. 3, pp. 977-983, 1988, doi: 10.1090/S0002-9939-1988-0947693-2.

[10] Z. Liu, R. P. Agarwal, and S. M. Kang, "On solvability of functional equations and system of functional equations arising in dynamic programming," J. Math. Anal. Appl., vol. 297, no. 1, pp. 111-130, 2004, doi: 10.1016/j.jmaa.2004.04.049.

[11] Z. Liu, "Coincidence theorems for expansion mappings with applications to the solutions of functional equations arising in dynamic programming," Acta Sci. Math., vol. 65, no. 1-2, pp. 359-369, 1999.

[12] Z. Liu, "Compatible mappings and fixed points," Acta Sci. Math., vol. 65, no. 1-2, pp. 371-383, 1999.

[13] Z. Liu, "Existence theorems of solutions for certain classes of functional equations arising in dynamic programming," J. Math. Anal. Appl., vol. 262, no. 2, pp. 529-553, 2001, doi: 10.1006/jmaa.2001.7551.

[14] Z. Liu and S. M. Kang, "Properties of solutions for certain functional equations arising in dynamic programming," J. Global Optim., vol. 34, no. 2, pp. 273-292, 2006, doi: 10.1007/s10898-0052605-6.

[15] Z. Liu and S. M. Kang, "Existence and uniqueness of solutions for two classes of functional equations arising in dynamic programming," Acta Math. Appl. Sin. Engl. Ser., vol. 23, no. 2, pp. 195-208, 2007, doi: 10.1090/S0002-9939-1969-0233341-4.

[16] Z. Liu and J. K. Kim, "A common fixed point theorem with applications in dynamic programming," Nonlinear Funct. Anal. Appl., vol. 11, no. 1, pp. 11-19, 2006.

[17] Z. Liu and J. S. Ume, "On properties of solutions for a class of functional equations arising in dynamic programming," J. Optim. Theory Appl., vol. 117, no. 3, pp. 533-551, 2003, doi: 10.1023/A:1023945621360.

[18] Z. Liu, J. S. Ume, and S. M. Kang, "Some existence theorems for functional equations arising in dynamic programming," J. Korean Math. Soc., vol. 43, no. 1, pp. 11-28, 2006.

[19] Z. Liu, Y. Xu, J. S. Ume, and S. M. Kang, "Solutions to two functional equations arising in dynamic programming," J. Comput. Anal. Appl., vol. 192, no. 2, pp. 251-269, 2006, doi: 10.1016/j.cam.2005.04.033.

[20] P. R. Meyers, “A converse to Banach's contraction theorem," J. Res. Nat. Bur. Standards, Sect B, vol. 71B, no. 2-3, pp. 73-76, 1967.

[21] M. Ohta and G. Nikaido, "Remarks on fixed point theorems in complete metric spaces," Math. Japon., vol. 39, pp. 287-290, 1994.

[22] H. K. Pathak and Deepmala, "Existence and uniqueness of solutions of functional equations arising in dynamic programming," Appl. Math. Comput., vol. 218, no. 13, pp. 7221-7230, 2012, doi: 10.1016/j.amc.2011.12.093.

[23] H. K. Pathak and B. Fisher, "Common fixed point theorems with applications in dynamic programming," Glas. Mat., vol. 31, pp. 321-328, 1996.

[24] S. P. Singh and B. A. Meade, "On common fixed point theorems," Bull. Aust. Math. Soc., vol. 16, no. 1, pp. 49-53, 1977, doi: 10.1017/S000497270002298X.

[25] S.-S. Zhang, "Some existence theorems of common and coincidence solutions for a class of functional equations arising in dynamic programming," Appl. Math. Mech. (English Ed.), vol. 12, no. 1, pp. 33-39, 1991, doi: 10.1007/BF02018064. 
Authors' addresses

\section{Guojing Jiang}

Basic Teaching Department, Dalian Vocational Technical College, Dalian, Liaoning 116035, People's Republic of China

E-mail address: jiangguojing@qq.com

\section{Shin Min Kang}

Department of Mathematics and The Research Institute of Natural Science, Gyeongsang National University, Jinju 660-701, Korea,

Center for General Education, China Medical University, Taichung 40402, Taiwan

E-mail address: smkang@gnu.ac.kr 